Homology, Homotopy and Applications, vol.11(2), 2009, pp.1-4

\title{
HOCHSCHILD HOMOLOGY WITH COEFFICIENTS IN AN $H$-UNITAL IDEAL
}

\author{
GURAM DONADZE AND MANUEL LADRA
}

(communicated by Jean-Louis Loday)

\begin{abstract}
Let $k$ be a field and $I$ an $H$-unital two-sided ideal of an associative unital $k$-algebra $A$. Then, based on Wodzicki's work, we show that the Hochschild homology groups of $A$ with coefficients in $I$ are isomorphic to the Hochschild homology groups of $I$.
\end{abstract}

\section{Definitions and conventions}

Let $k$ be a commutative unital ring, $A$ an associative unital algebra over $k$ and $M$ a bimodule over $A$. Consider the following complex, called the Hochschild complex,

$$
C(A, M):=\cdots \rightarrow M \otimes A^{\otimes n} \stackrel{b}{\longrightarrow} M \otimes A^{\otimes n-1} \stackrel{b}{\longrightarrow} \cdots \stackrel{b}{\longrightarrow} M \otimes A \stackrel{b}{\longrightarrow} M
$$

where $\otimes=\otimes_{k}$ and the Hochschild boundary $b: M \otimes A^{\otimes n} \rightarrow M \otimes A^{\otimes n-1}$ is the $k$-linear map given by the formula

$$
\begin{array}{r}
b\left(m, a_{1}, \ldots, a_{n}\right):=\left(m a_{1}, a_{2}, \ldots, a_{n}\right)+\sum_{i=1}^{n}(-1)^{i}\left(m, a_{1}, \ldots, a_{i} a_{i+1}, \ldots, a_{n}\right) \\
+(-1)^{n}\left(a_{n} m, a_{1}, \ldots, a_{n-1}\right) .
\end{array}
$$

By definition, the $n$th Hochschild homology group of $A$ with coefficients in $M$ is the $n$th homology group of $C(A, M)$ denoted by $H_{n}(A, M)$. In the case where $M=A$, the Hochschild complex is denoted by $C(A)$ and its $n$th homology is called the $n$th Hochschild homology group of $A$ denoted by $H H_{n}(A)$.

Let $I$ be a $k$-algebra (not necessarily unital). Denote by $I_{+}$the unital $k$-algebra $k \oplus I$, where the multiplication structure is given by

$$
\left(k_{1}, x\right)\left(k_{2}, y\right)=\left(k_{1} k_{2}, k_{1} y+x k_{2}+x y\right)
$$

The first author would like to thank the University of Santiago de Compostela for its hospitality during the work on this paper.

The second author was supported by MEC grant MTM 2006-15338-C02 (European FEDER support included) and by Xunta de Galicia grant PGIDITI06PXIB371128PR.

Received December 29, 2008; published on July 21, 2009.

2000 Mathematics Subject Classification: 17B40, 17B56, 18G10, 18G50, 18G60, $19 \mathrm{D} 55$.

Key words and phrases: Hochschild homology, $H$-unital algebra.

This article is available at http://intlpress.com/HHA/v11/n2/a1

Copyright (C) 2009, International Press. Permission to copy for private use granted. 
Then the $n$th Hochschild homology group of $I$ is defined as follows:

$$
H H_{n}(I):=H_{n}\left(\operatorname{Coker}\left\{C(k) \rightarrow C\left(I_{+}\right)\right\}\right) .
$$

Consider now the following complex, called the bar complex,

$$
C^{\text {bar }}(I): \cdots \rightarrow I^{\otimes n+1} \stackrel{b^{\prime}}{\longrightarrow} I^{\otimes n} \stackrel{b^{\prime}}{\longrightarrow} \cdots \stackrel{b^{\prime}}{\longrightarrow} I^{\otimes 2} \stackrel{b^{\prime}}{\longrightarrow} I,
$$

where the differential $b^{\prime}: I^{\otimes n+1} \rightarrow I^{\otimes n}$ is given by the formula

$$
b^{\prime}\left(x_{0}, x_{1}, \ldots, x_{n}\right):=\sum_{i=0}^{n-1}(-1)^{i}\left(x_{0}, x_{1}, \ldots, x_{i} x_{i+1}, \ldots, x_{n}\right) .
$$

Definition (M. Wodzicki). The $k$-algebra $I$ is said to be $H$-unital, if

$$
H_{*}\left(V \otimes C^{\mathrm{bar}}(I)\right)=0
$$

for any $k$-module $V$.

\section{Main result}

We prove the following:

Proposition 1. Let $k$ be a field, $A$ a unital $k$-algebra and $I$ an $H$-unital two-sided ideal of $A$. Then, for each $n \geqslant 0$, there are isomorphisms

$$
H_{n}(A, I)=H H_{n}(I) \quad \text { and } \quad H_{n}(A, A / I)=H H_{n}(A / I) .
$$

Proof. Consider the following commutative diagram of complexes:

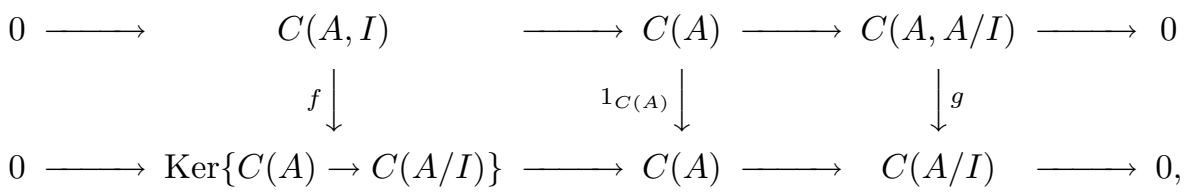

where $f$ and $g$ are naturally induced homomorphisms of complexes. We will show that $f$ and $g$ induce the isomorphisms:

$$
\begin{aligned}
& f_{*}: H_{n}(A, I) \stackrel{\cong}{\longrightarrow} H_{n}(\operatorname{Ker}\{C(A) \rightarrow C(A / I)\}), \\
& g_{*}: H_{n}(A, A / I) \stackrel{\cong}{\longrightarrow} H H_{n}(A / I),
\end{aligned}
$$

which will complete the proof, since, according to [2], there is an isomorphism

$$
H_{n}(\operatorname{Ker}\{C(A) \rightarrow C(A / I)\})=H H_{n}(I) .
$$

The upper row of (1) is exact, since $k$ is a field. By definition, the lower row of (1) is also exact. Thus, we have the following commutative diagram with exact rows:

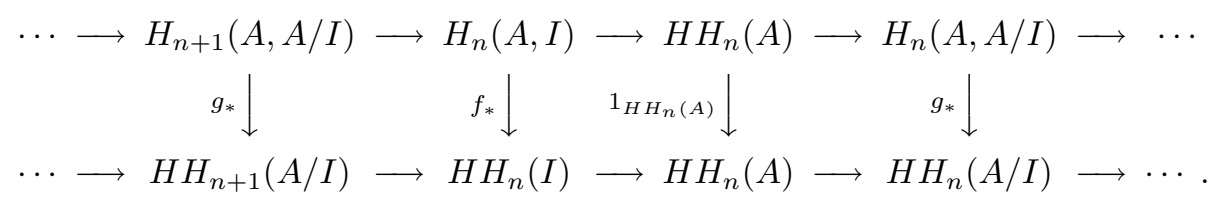

From (2) and by the Five Lemma it suffices to show that $g$ is a quasi-isomorphism. Since $g_{n}:(A / I) \otimes A^{\otimes n} \rightarrow(A / I) \otimes(A / I)^{\otimes n}$ is an epimorphism for each $n$, the key is to prove acyclicity for the complex Ker $g$. 
We need the following fact which is proved in [2] (see also [1]):

Lemma 1. Let $L=\operatorname{Ker}\left\{C^{\text {bar }}(A) \rightarrow C^{\text {bar }}(A / I)\right\}$. Define the following filtration on $L$ :

$F_{p} L_{n}=$ linear span of $\left\{\left(a_{0}, a_{1}, \ldots, a_{n}\right) \in L_{n} \mid\right.$ at least $n-p+1 a_{j}$ 's belong to $\left.I\right\}$.

Then the associated spectral sequence $E_{p q}^{r}(r \geqslant 0)$ is in the first quadrant and $E_{p q}^{1}=0$.

Return to the proof of Proposition 1. Define the following filtration on Ker $g$ :

$$
\begin{aligned}
\bar{F}_{p}\left(\operatorname{Ker} g_{n}\right)=\text { linear span of } & \left\{\left(a_{0}, a_{1}, \ldots, a_{n}\right)\right. \\
& \left.\in \operatorname{Ker} g_{n} \mid \text { at least } n-p a_{j} \text { 's }(j \geqslant 1) \text { belong to } I\right\} .
\end{aligned}
$$

Denote by $\bar{E}_{p q}^{r}(r \geqslant 0)$ the associated spectral sequence. We will show that $\bar{E}_{p *}^{1}=0$. By definition,

$$
\bar{F}_{p}\left(\operatorname{Ker} g_{n}\right)=(A / I) \otimes F_{p}\left(L_{n-1}\right) \Rightarrow \bar{E}_{p q}^{0}=(A / I) \otimes E_{p q-1}^{0},
$$

where $L, F$ and $E$ are defined as in Lemma 1 . Moreover, if $\left(a_{0}, \ldots, a_{n}\right) \in \bar{F}_{p}\left(\operatorname{Ker} g_{n}\right)$, then it is easy to check that

$$
\left(a_{0} a_{1}, a_{2}, \ldots, a_{n}\right) \quad \text { and } \quad\left(a_{n} a_{0}, a_{1}, \ldots, a_{n-1}\right) \in \bar{F}_{p-1}\left(\operatorname{Ker} g_{n-1}\right) .
$$

Thus,

$$
b\left(a_{0}, \ldots, a_{n}\right)=-a_{0} \otimes b^{\prime}\left(a_{1}, \ldots, a_{n}\right) \bmod \bar{F}_{p-1}\left(\operatorname{Ker} g_{n-1}\right) .
$$

Therefore, the following square

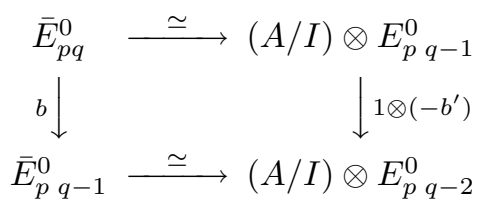

is commutative. Hence $\bar{E}_{p q}^{1}=(A / I) \otimes E_{p q-1}^{1}=0$.

Given a $k$-algebra $A$ (not necessarily unital) and an $A$-bimodule $M$, the complex $C(A, M)$ can be defined in the same way.

Let $I$ be a two-sided ideal of a $k$-algebra $A$. The inclusion $I \subset A$ induces the map of complexes $C(I) \rightarrow C(A, I)$ denoted by $\alpha_{(A, I)}$.

Proposition 2. Let $k$ be a field. Then the following are equivalent:

(i) $I$ is $H$-unital;

(ii) $\alpha_{(A, I)}$ is a quasi-isomorphism for any $k$-algebra $A$ which contains $I$ as a twosided ideal.

Proof of $(i) \Rightarrow$ (ii). Consider the following sequence:

$$
C(I) \stackrel{\alpha_{(A, I)}}{\longrightarrow} C(A, I) \stackrel{f}{\longrightarrow} \operatorname{Ker}\{C(A) \rightarrow C(A / I)\},
$$

where $f$ is naturally defined as in diagram (1). As in the previous proposition we can show that $f$ is a quasi-isomorphism, since in Lemma 1 it is not necessary to demand that $A$ is unital (see [1] or [2]). Moreover, by [2], $f \circ \alpha_{(A, I)}$ is a quasi-isomorphism. 
Proof of (ii) $\Rightarrow$ (i). We have to show that, if $\alpha_{(A, I)}$ is a quasi-isomorphism for any $A$, then $I$ is $H$-unital. We use the well-known trick. Namely, for any vector space $V$ consider the $k$-algebra $I \oplus V$ with multiplication given by $(x, v)(y, u)=(x y, 0)$. The quasi-isomorphism $C(I) \rightarrow C(I \oplus V, I)$ implies that $C(I \oplus V, I) / C(I)$ is acyclic. But $C(I \oplus k, I) / C(I)$ contains $V \otimes C^{\text {bar }}(I)[1]$ as a direct summand, where $C^{\text {bar }}(I)[1]$ means that the complex $C^{\text {bar }}(I)$ is shifted by 1 . Thus, $V \otimes C^{\text {bar }}(I)$ is acyclic.

Remark. In the case when $k$ is not a field, using the same arguments we can prove that a $k$-algebra $I$ is $H$-unital if and only if $\alpha_{(A, I)}$ is a quasi-isomorphism for any $k$-algebra $A$ which contains $I$ as a two-sided ideal and $A \rightarrow A / I$ is $k$-split.

Corollary 1. Let $k$ be a field and $A$ a unital algebra over $k$. If $I$ is an $H$-unital ideal of $A$, then for each $m \geqslant 1$ and $n \geqslant 0$, we have $H H_{n}\left(\mathcal{M}_{m}(A), \mathcal{M}_{m}(I)\right)=H H_{n}(I)$, where $\mathcal{M}_{m}(A)$ and $\mathcal{M}_{m}(I)$ denote the algebras of all $m \times m$ matrices with entries in $A$ and $I$, respectively.

Proof. Straightforward from Proposition 2 and [1, 1.4.14 Theorem].

Let $A$ be a unital algebra over $k$. Denote by $\overline{\mathcal{M}}(A)$ the algebra of all infinite matrices whose columns and rows contain a finite number of non-trivial elements. Put $\mathcal{M}(A)=\underset{m}{\lim } \mathcal{M}_{m}(A), m \geqslant 1$. Then $\mathcal{M}(A)$ is a two-sided ideal of $\overline{\mathcal{M}}(A)$ and we have the following:

Corollary 2. If $k$ is a field, then $H H_{n}(\overline{\mathcal{M}}(A), \mathcal{M}(A))=H H_{n}(A)$, for each $n \geqslant 0$.

Proof. It is known that $\mathcal{M}(A)$ is $H$-unital, since it has local units (see [1]). Hence $H H_{n}(\overline{\mathcal{M}}(A), \mathcal{M}(A))=H H_{n}(\mathcal{M}(A))$, but by Morita invariance we have

$$
H H_{n}(\mathcal{M}(A))=\underset{m}{\lim } H H_{n}\left(\mathcal{M}_{m}(A)\right)=\underset{m}{\lim _{m}} H H_{n}(A)=H H_{n}(A) .
$$

\section{References}

[1] J.-L. Loday, Cyclic homology, Grundlehren der Mathematischen Wissenschaften 301, Springer-Verlag, New York, 1992.

[2] M. Wodzicki, Excision in cyclic homology and in rational algebraic $K$-theory, Ann. of Math. (2) 129 (1989), no. 3, 591-639.

Guram Donadze donad@rmi.acnet.ge

A. Razmadze Mathematical Institute, Georgian Academy of Sciences, M. Alexidze St. 1, 0193 Tbilisi, Georgia

Manuel Ladra manuel.ladra@usc.es http://web.usc.es/ mladra

Departamento de Álgebra, Universidad de Santiago de Compostela, 15782 Santiago de Compostela, Spain 